\title{
The mental state of women with an IVF pregnancy
}

\author{
Vera A. Yakupova ${ }^{\mathrm{a}^{*}}$, Elena I. Zakharova ${ }^{\mathrm{a}}$, Ajdar N. Abubakirov ${ }^{\mathrm{b}}$ \\ ${ }^{a}$ Lomonosov Moscow State University, Moscow, Russia \\ ${ }^{b}$ Kulakov Scientific Center for Obstetrics, Gynecology and Perinatology, Moscow, Russia \\ ${ }^{\star}$ Corresponding author. E-mail: vera.a.romanova@gmail.com
}

\begin{abstract}
An in vitro fertilization (IVF) pregnancy is stressful both financially and emotionally. Patients undergoing an IVF procedure often have already had infertility and reproductive losses. Pregnancy through IVF involves the increased risk of various medical complications. Experts around the world are actively engaged in studying the specifics of the mental state of participants in IVF programs during pregnancy. Of critical importance is the issue of providing psychological support for couples who are preparing for and who have received an IVF pregnancy.

The aim of our research was to investigate the mental state of women participating in an IVF program. The study involved 224 pregnant women in the second and third trimesters: 62 women with an IVF pregnancy and 162 women who conceived naturally. The study took place at the Kulakov Scientific Center for Obstetrics, Gynecology, and Perinatology in Moscow, Russia. All the study participants had encountered medical complications during their pregnancy. No significant differences were identified in mental well-being in the two groups; this finding suggests that somatic complications during pregnancy are a general source of anxiety regardless of the reason for their occurrence. The second and third trimesters of pregnancy register increased anxiety levels associated with experiences of reproductive loss and the presence of physical problems. The main resources of a woman's personality that contribute to her self-confidence and mental stability are her professional employment and flexible behavior.
\end{abstract}

Keywords: IVF, psychology of pregnancy, mental state, motherhood

\section{Introduction}

IVF is becoming increasingly widespread, and many infertile couples are successfully becoming parents through its use. However, participation in an IVF program requires significant physical and psychological inputs. Preparing for IVF includes numerous surveys and intensive hormone therapy. Chances of success are initially about 50\% (Gardanova, 2008; Ola \& Ledger, 2005); with age the likelihood of pregnancy is reduced. Pregnancy is accompanied by constant risks because with IVF the chance of miscarriage, premature birth, and various somatic complications 
is higher than in a normal pregnancy (Lebedko, 2013; Sidelnikova, Khodjaeva, Strelchenko, Kiruschenkov, 2005).

We can confidently assert that the state of women with an IVF pregnancy is stressful. This stress is noteworthy because the mental state of a woman in pregnancy has a significant effect on its course and outcome (Zakharova \& Chuvaeva, 2011). Gardanova (2008) documents a high incidence of anxiety disorders among women preparing for IVF. Peak alarm occurs when they are taking a pregnancy test after IVF.

Swedish psychologists have studied women in the first trimester of their pregnancy and have found that the IVF group had a significantly higher level of anxiety associated with the risk of losing a child than the group with spontaneous conception. A long period of infertility contributes to higher anxiety (Hjelmstedt, Widstro, Wramsby, Matthiesen, \& Collins, 2003). There is evidence that this increased level of anxiety persists throughout the period of pregnancy. A study by Australian psychologists found that IVF women in their third trimester of pregnancy demonstrated higher levels of anxiety associated with the child's health and life than women who conceived naturally (McMahon, Ungerer, Beaurepaire, Tennant, \& Saunders, 1997). A Greek study of the coping strategies of women with an IVF pregnancy revealed a positive relationship between a problem-focused strategy and a favorable outcome for the IVF procedure (Gourounti et al., 2012). It also showed a heightened sense of lack of control over their own life, a feeling that increased with the number of IVF attempts.

So, when discussing the nature of anxiety among women pregnant after IVF, it can be assumed that the main source is their concern for their child's health and a favorable outcome for their pregnancy. One of the most important factors leading to increased anxiety is, in our opinion, the occurrence of physical complications during pregnancy. Because the probability of complications is higher among IVF women than among women who conceive naturally, we sought to equalize the sample studied according to this parameter. We selected women who conceived naturally and were in the hospital because of their physical problems to participate in the study as a comparison group. Thus, all the study participants, both those with IVF and those with natural conception, encountered medical complications during their pregnancy.

\section{The Study}

\section{Objectives}

The basic objective of the study was to compare the mental state of women with an IVF pregnancy and that of women who conceived naturally. In addition, we conducted an analysis of the possible causes of increased anxiety and the women's resources for coping with anxiety.

\section{Techniques}

We chose the Eysenck Personality Questionnaire (self-assessment of mental states) (Raigorodskiy, 2001) as a tool for assessing the mental state of pregnant women. The questionnaire consists of 4scales: Anxiety, Frustration, Aggression, and Rigid- 
ity. We also used the Emotional State scale of the Filippova questionnaire Style of Pregnancy Experiences (Filippova, 2002). The questionnaire suggests 6 styles of pregnancy experience: adequate, ambivalent, indifferent, euphoric, anxious, and resentful. For increased insight into pregnant women's psychology and possible sources of their anxiety we used the projective drawing technique Drawing of My Child and Me (Zakharova, 2014), in which each participant was asked to draw a picture of herself and her child.

\section{Sample}

The study was conducted at the Kulakov Center for Obstetrics, Gynecology, and Perinatology. The study involved 224 women in the second and third trimester of pregnancy at the Center's inpatient clinic, 162 women who had become pregnant with natural conception and 62 women who had become pregnant after IVF. All the study participants had encountered various somatic difficulties during their pregnancy and, as a result, found themselves in the clinic under medical supervision. The timing of the survey (the second and third trimester of pregnancy) was based on the fact that a woman's anxiety about continuing her pregnancy decreases during this period as she adapts to her condition, becomes aware of the change in her life, and feels her child's movements. The age of the women with natural conception (NC) ranged from 19 to 42; the average age was30.8. The age of the IVF women varied from 24 to 49 ; the average age was 33.2. In the NC group $32.4 \%$ (52 women) were not employed, $2.4 \%$ ( 4 women) were partially employed, and $65.2 \%$ (106 women) worked full-time. 1 of the unemployed women in this group quit her job after getting pregnant. The IVF group included $37.1 \%$ (23 women) unemployed, 1.6\% (2 women) partially employed, and 61.3\% (37 women) employed full-time. Of the unemployed women $39 \%$ (9) left work as soon as they conceived, significantly more than in the NC group (Fisher's exact test 4.192). In the NC group $62.3 \%$ (101 women) were nulliparous and $37.7 \%$ (61 women) were already mothers. In the IVF group $80.6 \%$ (50women) were nulliparous and $19.4 \%$ (12 women) were already mothers.

Of the study participants $31.6 \%$ did not provide any information about reproductive losses prior to the current pregnancy. In the NC group 48 women had experienced reproductive losses, 67 had no previous reproductive losses. In the IVF group 16 women had had a reproductive loss, while 22 had none.

The average waiting time for pregnancy in the NC group was 28 months, and it was 71 months in the IVF group.

\section{Results}

According to the data obtained using the Eysenck Personality Questionnaire (EPQ) the NC and IVF groups did not differ in level of anxiety: in both of them about one half of the respondents displayed a low level of anxiety, and a little less than one half showed an average anxiety level. Very few participants in the study rated their level on the Anxiety scale as being high. Thus, about half the respondents experienced anxiety, but very few of them had it in an intense form. A similar distribution of the results can also be observed on the Aggression, Frustration, and Rigidity scales (Table 1). 
Table 1.Distribution of responses on the EPQ scales in the NC and IVF groups

\begin{tabular}{|c|c|c|c|c|c|c|}
\hline & \multicolumn{3}{|c|}{ IVF Women } & \multicolumn{3}{|c|}{ NC Women } \\
\hline & $\begin{array}{l}\text { Low } \\
\text { level }\end{array}$ & $\begin{array}{c}\text { Average } \\
\text { level }\end{array}$ & $\begin{array}{l}\text { High } \\
\text { level }\end{array}$ & $\begin{array}{l}\text { Low } \\
\text { level }\end{array}$ & $\begin{array}{c}\text { Average } \\
\text { level }\end{array}$ & $\begin{array}{l}\text { High } \\
\text { level }\end{array}$ \\
\hline Anxiety & $50 \%$ & $45.2 \%$ & $4.8 \%$ & $48.4 \%$ & $47.3 \%$ & $4.3 \%$ \\
\hline Frustration & $68.7 \%$ & $27.4 \%$ & $3.9 \%$ & $70.5 \%$ & $25.9 \%$ & $3.6 \%$ \\
\hline Aggression & $46.6 \%$ & $50 \%$ & $3.4 \%$ & $48.7 \%$ & $46.7 \%$ & $4.6 \%$ \\
\hline Rigidity & $43.5 \%$ & $46.8 \%$ & $9.7 \%$ & $36.5 \%$ & $59.8 \%$ & $3.7 \%$ \\
\hline
\end{tabular}

These data suggest that the mental state of the women did not differ depending on their participation in an IVF program. The level of the severity of these conditions is not associated with a respondent's pregnancy stage or age.

Although the EPQ results showed that about half the women did not experience anxiety, the results of the projective technique Drawing of My Child and Me complemented the resulting picture by revealing their anxiety in a more graphic way. Most of the images for both groups contained signs of anxiety: abundance of shading, small pale figures, lack of color and clear lines in the drawing $(59 \%$ and $83 \%$ in the NC and IVF groups, respectively). At the same time, the IVF participants had significantly more alarming pictures (Fisher's exact test 1.84 at the 0.05 significance level) than did the NC women.

Thus, on the EPQ the answers of some of the women did not reflect the anxieties associated with their pregnancy. The women who had conceived after IVF appeared to be more inclined to disregard the difficulties that arose. Some studies suggest that a long infertility period may result in making a woman feel guilty if she «complains» about discomfort, difficulties, or moodiness (for example, Golombok, MacCallum, \& Goodman, 2001).

A reduction in anxiety and a disregard of difficulties that did arise were reflected in the Style of Pregnancy Experiences questionnaires. In both groups the pregnant respondents' emotional state can be described as ambivalent and indifferent; the distribution of responses in the groups did not differ significantly (Table 2).

Table 2.Distribution of responses for the NC and IVF groups on the Emotional State scale of the Style of Pregnancy Experiences questionnaire

\begin{tabular}{ccccccc}
\hline & Adequate & Anxious & Euphoric & Indifferent & Ambivalent & Resentful \\
\hline NC women & $17.8 \%$ & $2.5 \%$ & $13.3 \%$ & $21.5 \%$ & $40.5 \%$ & $4.4 \%$ \\
IVF women & $20 \%$ & - & $11.7 \%$ & $23.3 \%$ & $41.7 \%$ & $3.3 \%$ \\
\hline
\end{tabular}

Despite pregnancy complications both groups did not display severe anxiety. The IVF group had no alarm responses, while the NC group registered a mere $2.5 \%$ of anxiety responses. In contrast, the sample displayed a pronounced disregard for emerging problems: a large percentage of women deny that there have been any changes in their emotional status. The predominant answer in both 
groups was «I keep thinking about my own health and that of my child; I am afraid that something might happen to the baby during childbirth and that worries me a lot.» The Style of Pregnancy Experiences questionnaire rates this answer as ambivalent. Thus, our study confirms the findings of McMahon and colleagues (1997) that IVF program participants tend to worry about their child's health. However, our sample consisted of women who had experienced complications regardless of whether their pregnancy was the result of IVF. Thus, among women who faced real threats to a successful outcome of their pregnancy, concern for their child's health was heightened not only among those who became pregnant after IVF but also among those whose pregnancy was the result of natural conception.

This conclusion is confirmed by the discovery of a connection between anxiety level and previous reproductive losses. The women with no reproductive losses exhibited significantly lower anxiety levels than those with a history of miscarriage (Fisher exact test2.075 at a significance level of 0.05). Thus, previous experience of a spontaneous abortion contributes to anxiety, which remains pronounced throughout pregnancy. According to data obtained by Hjelmstedt and colleagues (2003), a high level of anxiety is associated with the risk of a miscarriage in the first trimester of pregnancy among IVF women. The most likely cause for increased levels of anxiety stems not only from a high risk of pregnancy termination among IVF women but also from the fact that IVF participants are more likely to have suffered numerous reproductive losses (Gardanova, 2008; Lebedko, 2013). This question should be researched at greater length as our study recorded only the fact that there were an unspecified number of reproductive losses in a woman's medical history.

Both groups under study revealed a similar pattern in the women's mental state. However, the IVF program participants, who had a long history of infertility (average 71 months), felt stronger and more resistant to failure than the NC women, who had tried to conceive a child for an average of 28 months. The longer the infertility period, the less frustration the respondents showed (Pearson's correlation coefficient 362 at the 0.01 significance level). The situation of infertility in the IVF participants may have become customary and have brought about a sense of habituation that reduced the level of their expectations and increased their willingness to meet with failure. Overcoming difficulties most likely contributes to the formation of self-reliance. Women who have overcome serious obstacles to motherhood and have achieved a long-awaited pregnancy may be more confident in their ability to resolve difficult situations in their life, are not afraid of difficulties, and find a way out of problematic situations. There is evidence that the feeling of being in control of a disease is positively related to psychological well-being (Leventhal \& Nerenz, 1980). The feeling of lack of control in the lives of couples facing infertility increases the level of stress (Litt, Tennen, Affleck, \& Klock, 1992). Most likely, women who have achieved the coveted pregnancy feel serene and confident. A study by Daniluk (2001) quotes couples speaking about their own personal growth and increased self-confidence after long treatment for infertility.

Overall, more than half the women surveyed in both groups experienced a low level of frustration. This result indicates that the sample consisted of fairly stable women who do not easily give in to difficulties, perhaps because of the relatively high socioeconomic status of the respondents. Level of frustration is related to 
employment and, consequently, to economic independence and education. In our sample, the higher a respondent's education level, the lower her level of frustration (Pearson's correlation coefficient 149 at a significance level of 0.05). The jobless women also experienced higher levels of frustration compared with the employed women (Pearson's correlation coefficient -189 at the 0.01 level). The study revealed that the unemployed women and poorly educated women alike felt powerless in the face of challenges as they found it difficult to overcome life's obstacles. Getting a higher education, to say nothing of a degree or a graduate-level education, involves overcoming many obstacles and takes much effort and willpower. The same goes for building a career. Women who seek no advances in the field of education and career may not feel strong and confident enough to overcome potential hurdles and subsequently rely on their partner, if they have one. Assumptions can be made about their personal characteristics, which affect both their career development and their ability to function as parents.

Overall, the sample revealed a positive association between the experience of motherhood and both self-confidence and a sense of one's own strength and resilience in the face of difficulties. The experienced mothers had an increased sense of self-competence, probably because motherhood promotes personal change. Moreover, the more children a mother had, the more confident she felt (Spearman's correlation coefficient -167 at a significance level of 0.05 ).

The employment rate relates not only to a sense of self-sustainability but also to behavioral flexibility. The women who quit their jobs the moment they got pregnant ( $4.5 \%$ of the sample, or 10 women, 9 of whom were IVF program participants) demonstrated a high level of rigidity in comparison with those who continued to work. Thus, the IVF program participants who put a sudden brake on their professional career found it difficult to adapt to change, to get used to new things. The failure to accept change in their life, to adapt to difficulties, may well constitute a psychological factor of their infertility.

A woman's ability to make effortless transitions and to behave flexibly changes with age. In the total sample, the distribution of scores on the Rigidity scale for different age groups was variable (according to the Mann-Whitney criteria, 037). In the older age group (37 and older) significantly fewer women had a pronounced level of rigidity; most possessed a low level. As they gain in life experience, women learn to adapt to change and become increasingly responsive and adaptable to sudden change in their lives.

Thus, we found no significant differences in the mental state of the women who conceived naturally and those participating in an IVF program.

\section{Conclusion}

First, the main source of the women's anxiety was not so much the fact of conception after IVF as the attendant physical problems; evidence for this conclusion was the fact that all the women in the NC group had medical complications. Thus, the mental state of women with physical problems during pregnancy does not differ significantly.

Second, when complications arose during pregnancy, the women tended to ignore them at the conscious level and to overcome their anxiety. On the unconscious 
level, the sample as a whole registered higher levels of anxiety. The results of the projective tests in the IVF group also showed higher anxiety levels. The IVF participants tended to be more inclined to overcome their anxieties than the women who conceived naturally.

Third, the main source of anxiety was previous reproductive losses and the presence of objective physical complications.

Fourth, the IVF women, who had a long infertility period, became accustomed to a situation of failure; as a consequence, they exhibited greater stability and willingness to fight for their goals than the NC women, who had shorter periods of infertility. Internal resources that contributed to the women's sense of self-confidence included professional engagement and behavioral flexibility, which makes it possible to successfully adapt to change.

\section{Acknowledgments}

We express our gratitude to the leadership of the Kulakov Scientific Center for Obstetrics, Gynecology, and Perinatology (a federal budgetary institution) for assistance in organizing this study.

\section{References}

Daniluk, J. C. (2001). "If we had it to do over again ... ": Couples' reflections on their experiences of infertility treatments. Family Journal, 9, 122-133 doi: 10.1177/1066480701092006

Filippova, G.G. (2002). Psychology of Motherhood: Textbook. Moscow: Institute of Psychotherapy.

Gardanova, Zh.R. (2008). Borderline mental disorders in women with infertility in assisted reproductive technology programs and their psychotherapeutic correction (Unpublished doctoral dissertation). Moscow: Moscow Medical and Stomatological Institute.

Golombok, S., MacCallum, F., \& Goodman, E. (2001).The "test-tube" generation: Parent-child relationships and the psychological well-being of in vitro fertilization children at adolescence. Child Development, 72(2), 599-608. doi: 10.1111/1467-8624.00299

Gourounti, K., Anagnostopoulos, F., Potamianos, G., Lykeridou, K., Schmidt, L., \& Vaslamatzis, G. (2012). Perception of control, coping and psychological stress of infertile women undergoing IVF. Reproductive BioMedicine Online, 24(6), 670-679. doi: 10.1016/j. rbmo.2012.03.002

Hjelmstedt, A., Widstro, A. M., Wramsby, H., Matthiesen, A. S., \& Collins, A. (2003). Personality factors and emotional responses to pregnancy among IVF couples in early pregnancy: A comparative study. Acta Obstetricia et Gynecologica Scandinavica, 82, 152-161. doi: 10.1034/j.1600-0412.2003.00040.x

Lebedko, A. V. (2013). Special aspects of pregnancy and childbirth among IVF women. (Abstract of master's thesis). Minsk: Belarusian State Medical University.

Leventhal, H., Meyer, D., \&Nerenz, D. (1980). The common sense representation of illness danger. In S. Rachman (Ed.), Medical psychology (vol. 2, pp. 7-30). New York: Pergamon Press.

Litt, M., Tennen, H., Affleck, G., \& Klock, S. (1992). Coping and cognitive factors in adaptation to in vitro fertilization. Journal of Behavioral Medicine, 15, 171-187. doi: 10.1007/BF00848324

McMahon, C. A., Ungerer, J. A., Beaurepaire, J., Tennant, C., \& Saunders, D. (1997). Anxiety during pregnancy and fetal attachment after in-vitro fertilization conception. Human Reproduction, 12, 176-182. doi: 10.1093/humrep/12.1.176 
Ola, B., \& Ledger, W. L. (2005). In vitro fertilization. Current Obstetrics \& Gynaecology, 15, 314323. doi: 10.1016/j.curobgyn.2005.06.006

Raigorodskiy, D. Ya. (Ed.). (2001). Practical psychodiagnostics. Techniques and tests. A teaching aid. Samara, Russia: Bakhrakh-M.

Sidelnikova, V. M., Khodjaeva, Z. S., Strelchenko, M. B., Kiruschenkov P. A. (2005). Peculiarities and tactics of pregnancy management after IVF. In V.I. Kulakov, B.V. Leonov, \& L.N. Kuzmicheva (Eds.), Treatment of female and male infertility. Assisted reproductive technologies. (pp. 574-579). Moscow: Russian News Agency.

Zakharova, E. I. (2014). Psychology of parenthood development. Moscow: IIU MGOU.

Zakharova, E. I., \& Chuvaeva, A. S. (2011). Psychological aspects of recurrent miscarriage. Journal of Obstetrics and Women's Diseases, 40, 41-42.

Original manuscript received September 24, 2014

Revised manuscript accepted December 23, 2014

First published online March 31, 2015 